\title{
How to get doctors to hand hygiene: nudge nudge
}

\author{
A Kwok ${ }^{*}$ M-L McLaws \\ From 3rd International Conference on Prevention and Infection Control (ICPIC 2015) \\ Geneva, Switzerland. 16-19 June 2015
}

\section{Introduction}

The Nudge Theory has been widely used to improve healthy behavior $[1,2]$ and works as an external cue to memory [3]. The Nudge Theory is based on behavioural economics models that has been applied to move communities towards rational targeted purchasing and ecological preferred behaviour patterns [1,2]. Consumers have been nudged successfully towards lower electricity purchasing patterns through displays of their past and current purchasing pattern.

\section{Objectives}

To engage medical staff towards improved hand hygiene compliance.

\section{Methods}

An automated hand hygiene surveillance system was installed in an Australian tertiary teaching hospital. The clinicians were taught to access a dashboard for daily compliance rates to be discussed at hand-over meetings and to use a seven-step approach that included nudging each other on the wards with "Doctor, take a moment". Feedback from clinicians about nudging and rates on both wards from June 2014 to February 2015 were compared.

\section{Results}

During the run-in period prior to introducing nudging the baseline compliance rate was $18 \%$ on ward $C$ and $37 \%$ on ward D. Preliminary results indicated one ward has improved by 32 percentage points while compliance on ward C remained stable at $15 \%$. Clinicians on ward $D$ reported that they were comfortable working as a team to nudge each other towards a goal of improved daily compliance and it was fun. Conversely, ward C clinicians reported a discomfort with nudging each other and were observed to have a different ward culture than ward D. We will discuss the difference in the staff and leadership on the wards that may explain the opposing attitudes towards nudging.

\section{Conclusion}

Nudging daily compliance rates by nurses and doctors resulted in collegiality and developed a 'team consciousness' about improvement in compliance. Changing hand hygiene compliance must work with different organization cultures on wards for effective change management.

\section{Disclosure of interest}

A. Kwok Conflict with: Deb Australia, Debgroup UK provided the automated system to collect daily compliance rates. The authors declared have no other conflict of interests. , M.L. McLaws Conflict with: Deb Australia, Debgroup UK provided the automated system to collect daily compliance rates. The authors declared have no other conflicts of interest.

Published: 16 June 2015

\section{References}

1. Thaler R, Sunstein C: Nudge: Improving decisions about health, wealth and happiness. Penguin Books Ltd UK; 2009.

2. Vallgarda S: Nudge- A new and better way to improve health? Health Policy 2012, 104(2):200-203.

3. Sax H, Clark L: . J. Hospital Infect 2015, 89(4):335-339.

doi:10.1186/2047-2994-4-S1-051

Cite this article as: Kwok and McLaws: How to get doctors to hand hygiene: nudge nudge. Antimicrobial Resistance and Infection Control 20154 (Suppl 1):051. 